\title{
L'HISTOIRE, LA FABLE ET LE FABULEUX ANALYSE DE LA NOTION DE FABULEUX
}

\author{
Nicolas PIQUÉ
}

\begin{abstract}
Résumé: Paul Hazard a très bien montré la concomitance de la Crise de la conscience européenne dans divers domaines, sous divers aspects et divers questionnements. Cette crise, ces nouvelles problématiques qui surgissent concernent également la conception de la temporalité qui avait cours jusqu'alors. Nous aimerions, dans cet article, en montrer l'émergence à partir d'une question particulière, qui concerne le statut et la signification à accorder aux fables, aux mythes que nous avons hérités de l'Antiquité. Grâce à l'étude que mènent l'abbé Anselme, Fontenelle, Nicolas Fréret, le chevalier de La Barre et Lévesque de Burigny, de la notion de merveilleux, comme contenu des fables, nous pourrons mieux saisir le développement d'une pensée de l'histoire.
\end{abstract}

Mots-CLÉs : histoire, fable, xvII ${ }^{e}$ siècle, Antiquité, herméneutique.

ABSTRACT: Paul Hazard has clearly shown the concomitance of The Crisis of the European consciousness in various domains, under diverse aspects and diverse interrogations. This crisis, these new issues which arise concern as well the concept of temporality which was then in vogue. This article seeks to study its emergence through a specific question which concerns the status and the significance to be given fables and myths which we have inherited from Antiquity. Thanks to the studies by abbé Anselme, Fontenelle, Nicolas Fréret, the chevalier de La Barre and Lévesque de Burigny of the notion of the marvelous, as found in fables, we can better grasp the development of an historical concept.

KEYWORDS : history, tale, 18th century, Antiquity, hermeneutics.

Revue de synthèse : $4^{e} \mathrm{~S} . \mathrm{N}^{\mathrm{o}} 1$, janv.-mars 1997, p. 65-81. 
Zusammenfassung: Paul Hazard hat unter verschiedenen Aspekten und Fragestellungen auf das gleichzeitige Auftreten einer Krise des europäischen Bewußtseins in unterschiedlichen Bereichen hingewiesen. Bei den mit dieser Krise zusammenhängenden Problemen geht es auch um den Begriff der Zeitlichkeit. In diesem Artikel sollen die Funktion und die Bedeutung der Fabeln und Mythen untersucht werden, die aus der Antike überliefert sind. Anhand der von abbé Anselme, Fontenelle, Nicolas Fréret, dem chevalier de La Barre und Lévesque de Burigny durchgeführten Untersuchungen zum Begriff des Wunderbaren in den Fabeln läßt sich die Entwicklung einer bestimmten Form des geschichtlichen Denkens erfassen.

STICHWÖRTER: Geschichte, Fabeldichtung, 18. Jahrhundert, Altertum, Hermeneutik.

Nicolas Piqué, né en 1966, ancien élève de l'École normale supérieure de Fontenay/SaintCloud, travaille sur l'histoire aux XVII et XVIII ${ }^{e}$ siècles, particulièrement à travers les controverses théologiques.

Adresse : CERPHI, ENS Fontenay, 31 av. Lombart, 92260 Fontenay-aux-Roses. 
LA FABLE ET LE FABULEUX : ENTRE AFFABULATION ET RECONNAISSANCE

Au début du $\mathrm{XVIII}^{\mathrm{e}}$ siècle, la connaissance des fables est nécessaire pour comprendre la culture de l'époque. Des références aux fables peuplent et structurent l'ensemble des productions culturelles, de la peinture à la littérature, en passant par la sculpture ou encore l'opéra. Qui ne sait reconnaître les allusions, les références sera incapable de décrypter ce que son époque lui propose et lui présente.

Rien d'étonnant donc à ce que Charles Rollin, dans son Traité des études, marque le caractère essentiel d'un tel savoir. L'intérêt pour les fables relève donc d'un nécessaire entraînement à un jeu de décodage, comme le remarque Jean Starobinski ${ }^{1}$. C. Rollin ne se prononce pas tant pour les fables elles-mêmes que pour l'utilisation qui en est faite et qui rend nécessaire une familiarisation, que les études ont, entre autres choses, pour mission de favoriser.

Mais, dans le même mouvement, on peut remarquer que les fables comportent également une dimension étrange, mystérieuse et merveilleuse, qui heurte les lecteurs du XVIII ${ }^{e}$ siècle. À tel point que C. Rollin précise comment mettre à distance cet aspect des fables : « Cette étude, quand elle est faite avec les précautions et la sagesse que demande et qu'inspire la religion, peut être d'une très grande utilité pour les jeunes. " Il faut faire attention de procéder avec «sagesse et précaution ${ }^{2}$.

On pourrait retrouver cette double appréciation des fables dans le Dictionnaire de la fable de Pierre Chompré ou encore dans l'article « Fable » de l'Encyclopédie, article dû à Louis Jaucourt. Comment lire les fables tout en se méfiant de certains détails qu'elles rapportent? On retrouve cette double dimension dans la notion de fabuleux. Le fabuleux est d'abord ce que la fable renferme. Mais c'est également ce qui heurte le sens commun du xvIII' siècle, et qu'il faut rejeter.

Ainsi, si l'on lit les fables à cette époque, on ne s'interdit nullement d'étudier de manière critique le fabuleux, au moins de le mettre à distance. Comment le comprendre, quelle place lui faire? Quelle signification lui donner? Cette question nous semble permettre de mieux apprécier le rap-

1. Jean StarobinSKI, «Le mythe au XVmi siècle », Critique, 7, 1977, p. 975-997.

2. Charles Roluin, Traité des études, Paris, 1726-1728, liv. VI, $4^{\mathrm{e}}$ part. 
port entre le $x^{\prime}{ }^{\mathrm{e}}$ siècle et l'Antiquité, le rapport que cette époque entretient avec l'histoire.

En effet, l'attitude à l'égard du fabuleux, du contenu des fables principalement grecques et latines, détermine une certaine conception de l'histoire. Reconnaître au fabuleux une signification propre, digne d'intérêt parce qu'informant notre connaissance de l'histoire ou, au contraire, voir dans le fabuleux le résultat de l'affabulation des poètes (assimilant par là le fabuleux et l'erreur, jugeant de ce fait du fabuleux par rapport au critère de vérité), ces deux conceptions renvoient à deux visions de l'histoire. Plus précisément même, nous pourrons voir dans cette différence d'approche la ligne de partage entre une pensée de l'Histoire et un discours de la Tradition.

\section{LA PLACE DU FABULEUX DANS UN PROJET APOLOGÉTIQUE}

Le père Anselme fut membre de l'Académie des inscriptions et belleslettres ${ }^{3}$ comme presque tous les savants qui s'intéressaient à l'histoire des peuples de l'Antiquité. Il y participa à la querelle à propos de la fiabilité des documents concernant l'histoire antique ${ }^{4}$, durant laquelle il s'opposa à Louis-Jean Lévesque de Pouilly, dont nous aurons l'occasion de reparler. Mais c'est une autre de ses communications à cette académie qui nous intéresse.

Elle s'intitule «Dissertation sur ce que le paganisme a publié de merveilleux ${ }^{5} »$. Ce texte est tout à fait révélateur d'une manière d'analyser le fabuleux, le «merveilleux » pour le père Anselme. Modalité spécifique, parfaitement rodée, et largement utilisée au XvIII ${ }^{\mathrm{e}}$ siècle ${ }^{6}$.

3. Désormais appelée AIBL dans les notes.

4. «Monuments qui ont suppléé au défaut de l'écriture, et servi de Mémoires aux premiers historiens », Mémoires de l'AIBL, t. IV, Paris, 1723.

5. Elle fut lue, conformément à l'usage, en séance publique le 6 avril 1717 et publiée en 1723 dans le tome IV des Mémoires de ladite académie.

6. L'étude par le père Anselme des fables grecques et latines, ainsi d'ailleurs que des documents d'autres civilisations, se fait au sein d'un projet, sinon explicitement apologétique, du moins respectant les enseignements dogmatiques de la religion chrétienne et les renseignements historiques de la Bible. Il s'agit d'expliquer, et non de comprendre, ces fables à partir du cadre historique fourni par la Bible. Ce cadre chronologique défínit les limites et les dates essentielles de l'Antiquité de l'humanité entière. Il impose de ce fait une lecture historique, qui favorise l'unité, la continuité et la globalité. Il ne saurait être question que des événements, ou a fortiori des civilisations entières, puissent échapper à l'étude que rend possible la Bible. De là provient la formulation de la question de l'apologie historique, telle que nous allons l'étudier. Le père Anselme met en œuvre ce modèle, sans en être le seul représentant. On pourrait citer Guillaume de LAvaur, Conférence de la fable avec l'Histoire sainte, Paris, 1730; l'abbé Michel Fourmont, «Explication de la fable d'Orion, dans laquelle on la rappelle à l'Histoire sainte », Mémoires de l'AIBL, Paris, 1743; l'abbé Louis-François DE FonTENU, « Réflexions sur l'utilité que les belles-lettres peuvent tirer de l'Écriture sainte », Mémoires de l'AIBL, 
La dissertation révèle tout d'abord une critique non voilée des fables et du merveilleux qu'elles véhiculent. L'étude du vocabulaire utilisé est symptomatique à cet égard. Le père Anselme décrit le merveilleux grâce aux notions suivantes : il parle des « obscurités » que colportent les fables, des « traditions fabuleuses » qui ont pu abuser la « crédulité » de certains, pour conclure que les «prédictions et les prodiges tant vantés dans l'Antiquité païenne n'ont été que des impostures ». Décrire ce que recèlent les fables ne peut se faire que par l'entremise du vocabulaire de l'erreur, voire de la manipulation. Le père révèle, en effet, que ces fables ont été construites, fabriquées par ceux qui pensaient grâce au « merveilleux flatt[er] la vanité des peuples parce qu'un événement singulier suffisait pour rendre un pays célèbre ».

Toutefois nous, «qui avons des lumières plus pures », ne sommes pas dupes de ce merveilleux. Mais, et c'est une des particularités de ce texte, au sein de l'Antiquité, certains, les plus sages, avaient déjà attiré l'attention sur le caractère peu crédible de ce merveilleux. Et le père Anselme de citer Platon, Varron, Plutarque, Pline. Seuls les stoïciens, parce que cela s'accordait trop avec leur vision du destin, ont toujours tenu ce fabuleux pour vrai. L'appréciation de l'Antiquité, à travers ses productions culturelles et leur principale caractéristique, le merveilleux, est donc particulièrement subtile. Elle n'est pas positive, puisqu'elle relève avant tout de l'erreur. Mais dans le même mouvement, le père Anselme la sauve, en clivant les productions: Au sein même de l'Antiquité, on peut identifier des pôles qui permettent de justifier l'attrait pour cette période. Ce sauvetage permet de comprendre pourquoi son analyse ne se termine pas par le rejet pur et simple de ces textes hors de sa sphère d'intérêt, dont la cohérence est chrétienne avant d'être rationnelle.

Les raisons d'une telle sauvegarde de l'intérêt pour les fables antiques pourraient n'être que simplement sociologiques : comment justifier au $\mathrm{XVIII}^{\mathrm{e}}$ siècle des pratiques immémoriales en les mettant en accord avec les nouvelles exigences de rationalité? Il nous semble en fait que le schéma d'analyse du père Anselme est guidé par un tout autre enjeu. Dans un projet apologétique de type continuiste ${ }^{7}$, il n'est pas possible de ne pas essayer d'intégrer toute une période dans le cadre de justification que constitue ce projet. Surtout quand cette période occupe une place aussi stratégique.

Paris, 1744. Parallèlement à ce projet apologétique, s'est développée une étude critique de la chronologie sacrée, à partir notamment des chronologies chinoises, mais aussi de calculs astronomiques, dont les promoteurs sont Jules César Scaliger et Gerardus Johannis Vossius, mais aussi Nicolas Fréret. Le projet du père Anselme ne tient aucun compte de ces nouvelles approches.

7. Nous avons déjà indiqué le lien qui existe entre le type de lecture de l'histoire que l'on peut faire à partir de la Bible, et le caractère nécessairement globalisant, et donc continuiste, d'une telle vision de la temporalité. La matrice que représente la Bible ne saurait laisser subsister des périodes à l'extérieur du cadre qu'elle définit. 
En effet, du point de vue du père Anselme, l'Antiquité gréco-latine appartient à son aire, aussi bien culturelle qu'historique. La question se pose dès lors de savoir comment rendre compte de cet îlot qui semble $a$ priori devoir échapper aux schémas de lecture de l'histoire de l'humanité que propose la Bible. Il n'est pas possible de laisser de côté cette période, le père Anselme et ses contemporains en sont trop évidemment les héritiers, comme nous l'avons remarqué plus haut par l'intermédiaire de la production culturelle; à la différence de la Chine ou de l'Amérique, qu'il est bien plus facile d'écarter de ce discours apologétique nécessairement globalisant. De plus, les sociétés antiques ont souvent été présentées comme l'origine de l'Europe. Or l'origine ne peut être ailleurs qu'en Terre sainte comme l'indique la Bible. Il faut par conséquent absolument intégrer cette page de notre histoire au sein du cadre chronologique ou ontologique que constitue toute apologie historique. Et s'il était possible de l'y intégrer, il devient dès lors possible de faire fonctionner une logique de la dérivation qui montrera comment la Grèce et Rome peuvent être insérées dans ce cadre. Le merveilleux n'a par conséquent de sens que s'il est intégré dans cette logique de la dérivation, dont nous proposons de mettre en évidence le fonctionnement.

L'analyse du merveilleux antique doit donc satisfaire à deux exigences. D'une part, ne pas laisser de côté la production culturelle d'une période, dont la place est si évidemment stratégique. Mais, d'autre part, ne pas laisser subsister tel quel le contenu merveilleux des fables. Le père Anselme propose une analyse qui répond à ces deux critères.

Ainsi, même au plus fort de la critique, le prestige de l'Antiquité doit être sauvegardé. De telle sorte que ce ne sont pas les peuples de l'Antiquité qui doivent être tenus pour responsables de ce merveilleux, qui heurte toute la raison, mais certains vaniteux qui ne pouvaient se passer de signes merveilleux, appelés à justifier l'importance de leur société ${ }^{8}$.

Toutefois, l'admiration de l'Antiquité est-elle le seul motif d'un tel sauvetage? Ne pourrait-on pas y lire également la volonté de garder cette période dans le cadre d'une simple erreur vis-à-vis de la « vraie Religion »; laisser penser que le paganisme aurait régné positivement est une thèse

8. Cette notion de « vanité " est tout à fait intéressante; le père Anselme, on l'a vu, parle de la " vanité des peuples ». La notion caractérise la volonté qu'a chaque peuple de se trouver des origines valorisantes, et donc en partie fabuleuses. Charles de Saint-Évremond, dans Réflexions sur les divers génies du peuple romain (1663), est un des premiers à utiliser ce concept critique, que nous retrouverons également chez Fontenelle et Jean Lévesque de Burigny, et que Giambattista Vico consacrera dans la Science nouvelle (1725). Il faut remarquer la conception du temps qu'implique cette recherche d'origines vaniteuses. Chercher à valoriser ses origines n'a de sens que si l'on pense que l'origine est également fondement substantiel. La vanité des origines recouvre donc le postulat suivant: l'origine n'est pas un simple commencement, la plénitude est initiale et le temps n'amène aucune nouveauté. Le temps n'est pas créateur, il impose une unification, qui trouve son fondement dans l'origine. 
qu'il est bien plus difficile de comprendre d'un point de vue apologétique. En effet, reconnaître une indépendance au paganisme et au merveilleux qu'il véhicule, reviendrait à laisser l'Antiquité à l'écart de l'influence divine. Si le paganisme avait existé positivement comme tel, cela reviendrait à lui reconnaître une antériorité chronologique, et donc ontologique, sur le christianisme. En effet, pour qui pense que l'histoire se joue dans l'origine, l'antériorité chronologique équivaut à une priorité quant à la vérité.

Car le fond de l'argumentation du père Anselme reste bien apologétique, quoiqu'il s'en défende ( nous ne faisons pas ici une apologie de la religion chrétienne »). Et, au sein de ce projet, il lui faut sauver l'Antiquité d'un rejet du côté de la simple insignifiance, car elle va lui servir de pierre de touche dans le cadre d'une logique de la dérivation. Il est possible, pour nous qui sommes du côté de la « vraie Religion », de comprendre les fables au-delà des affabulations ${ }^{9}$ des prêtres du paganisme. Il suffit de les faire dériver « du plus vrai comme du plus ancien livre du monde, où il est probable que les plus éclairés des païens avaient pensé ce que l'on trouve de plus sensé ».

Ainsi, étant entendu que «nous savons que les prédictions et les miracles ne pouvaient venir que de Dieu ", il devient nécessaire d'opérer une série de distinctions : entre les "païens habiles et sages" et les prêtres ainsi qu'entre ce que les fables renferment de sensé et le merveilleux résultat d'affabulation vaniteuse. De telle sorte que l'Antiquité, et l'admiration qu'elle suscite malgré tout, est légitimée en servant même d'arguments apologétiques. Les fables sont des signes, déguisés, dérivés, de la «vraie Religion ». Et l'un des buts de l'Académie des inscriptions et belles-lettres « dont l'objet est de dévoiler l'Antiquité la plus éloignée » consistera donc à regarder « les livres saints comme nécessaires pour bien comprendre l'histoire de tous les temps ». On comprend ainsi la nécessité, affirmée avec force, de ne pas voir l'Antiquité comme un bloc et de pratiquer une série de distinctions. Le merveilleux relève de l'erreur, mais comme il est une production de l'Antiquité, il devient possible, grâce à ces distinctions, de sauver cette Antiquité, origine à préserver, et même de s'en servir dans une perspective chrétienne.

Il va donc s'agir de «dévoiler » la vérité, ce que seule permet cette logique de la dérivation, qui consiste à retrouver dans les fables, y compris le fabuleux, des signes, des reprises de la Bible. Il devient alors possible de « connaître l'histoire de tous les temps ». Il faut prendre, nous semble-t-il,

9. Le terme n'est pas explicitement présent chez le père Anselme, alors qu'il l'est par exemple chez le père Tournemine. Toutefois, l'analyse que mène le père Anselme du merveilleux en terme d'erreur, par rapport à la religion, comme nous l'avons montré, autorise cet emploi. Ce qui permet de bien caractériser l'enjeu de son étude. 
cette expression à la lettre. Tout ce que l'histoire des différents temps, des différentes époques pourra nous apprendre, nous pourrons le retrouver dans la Bible, et grâce à elle. Ce schéma herméneutique circulaire, chercher à justifier la religion (donc la véracité de la Bible) dans des témoignages qui ne deviennent signifiants que dans une référence à la Bible, nous pouvons le caractériser comme tradition ${ }^{10}$. Ainsi l'unité du temps est sauvée. Elle l'est en niant l'histoire (si l'on entend par histoire une conception du temps qui accorde une signification propre à chacune de ses parties, sans que le sens de la totalité du devenir temporel ne soit fixé dans une origine anhistorique), mais en intégrant, en tenant compte des témoignages du passé.

Il n'en demeure pas moins que ces témoignages, le merveilleux en ce qui concerne l'Antiquité, n'existent et ne prennent sens que par rapport à quelque chose qui leur est totalement étranger : la Bible. Tel est le sens de la logique de la dérivation. Chaque société n'est jamais que la modélisation, nullement essentielle, nullement autonome, d'une vérité qui la transcende entièrement. Et ce n'est que grâce à nos « lumières plus pures » que nous arrivons à transformer l'erreur du paganisme en vérité chrétienne. Il était toutefois utile, dans cette optique, d'inscrire au sein même du paganisme cette dualité (que symbolise l'écart qui sépare les plus sensés des païens des autres, qui sont même accusés d'user de « ruses »). Tant il est vrai qu'il vaut mieux avoir affaire à un paganisme dévoyé dans une erreur qu'à un paganisme entièrement autonomisé vis-à-vis de la religion chrétienne.

Dans cette dissertation du père Anselme, nous avons essayé de mettre en évidence différents schémas d'analyse de l'histoire, ou plutôt du temps, et on a pu en étudier le fonctionnement à propos de la notion de merveilleux. L'utilisation du vocabulaire de l'erreur, de l'imposture et donc de l'affabulation dans le cadre d'une analyse du merveilleux rend possible l'application de la logique que nous avons décrite. L'Antiquité païenne n'échappe pas à la vérité chrétienne, elle en est simplement l'image déformée, renversée $^{11}$, qu'il est parfaitement, et simplement, possible de remettre à l'endroit. En prenant conscience que la Bible, le plus ancien donc le plus

10. Si l'on définit la tradition comme un discours des origines. L'origine constitue alors le foyer atemporel du sens de la réalité, au-delà de toute inscription historique spécifique. Le discours de la tradition s'oppose alors à une pensée de l'histoire, comme nous allons le voir, l'histoire ne pouvant véritablement advenir que sur les ruines de l'origine.

11. Il peut être intéressant de remarquer la postérité philosophique de cette notion de renversement. Hegel, Marx penseront pouvoir faire apparaître le sens de la réalité, à partir d'un renversement dialectique. L'enjeu reste le même : trouver dans une matrice le principe herméneutique qui permettra de révéler le sens plein de la réalité. Bien entendu, le père Anselme ne cherche nullement à fonder de manière dialectique cette relation. En revanche, au sein même de la période qui nous intéresse, d'autres ont remis en cause la possibilité même d'une telle lecture. Le chevalier de La Barre remarque, en effet, dans ses « Mémoires pour servir à l'histoire de la religion de la Grèce », Mémoires de l'AIBL, t. XVI, Paris, $1751:$ «...] il a fallu renverser les fables pour y retrouver l'Histoire sainte, avec une licence qui n'a point eu de bornes. On a usé des fables comme des mots d'une langue pour exprimer tout ce qu'on veut. » 
vrai des livres, est la matrice de toute signification possible. Tout est donné dans la Bible; pour qui sait lire l'histoire du monde, qui dès lors n'en est plus vraiment une, ou plutôt les histoires du monde, cette vérité apparaîtra clairement. Ce figurisme mythologique ${ }^{12}$ aboutit donc à une éviction de l'histoire. Le fabuleux n'est que le signe de la nécessité de trouver le sens des fables, la vérité des fables dans le rapport à l'origine de toutes vérités, de tous les temps : la Bible.

On pourrait retrouver dans de nombreux autres textes ces mêmes préoccupations, ces mêmes schémas de pensée. Traiter du fabuleux en termes de vérité et d'erreur est le centre du travail du père Tournemine par exemple. Lire les fables, se trouver confronté au fabuleux n'a de sens qu'à vouloir y débusquer l'erreur. Le fabuleux n'a plus alors d'intérêt en luimême. Passer de l'erreur à la vérité, c'est-à-dire établir le rapport entre les fables et la religion chrétienne, se fait par l'intermédiaire d'une logique de la dérivation. Celle-ci est mise en œuvre grâce à des procédés étymologiques et d'analogies de structures narratives. Le père Huet, bien entendu, mais également les collègues du père Anselme à l'Académie des inscriptions et belles-lettres, que sont Guillaume de Lavaur, Jean-Baptiste Souchay et Joseph-Balthasar Gibert, se sont faits par ailleurs également les hérauts d'une telle recherche.

\footnotetext{
Le chevalier de La Barre critique cette façon d'interpréter les fables; nous verrons plus loin quels sont les principes qu'il propose.

12. Le figurisme est cette pratique exégétique, remontant à l'exégèse médiévale (cf. Henri DE Lubac, Exégèse médiévale. Les quatre sens de l'Écriture, Paris, Montaigne/Aubier, 19591964, et ID., «Typologie et allégorisme », Revue des sciences religieuses, 34, 1947), qui consiste à adopter comme principe de lecture de la Bible la règle herméneutique suivante : l'ensemble de la Bible, des péripéties et histoires qu'elle rapporte, prend sens par rapport à la figure du Christ. Cette exégèse fut reprise et amplifiée à Port-Royal, où elle fut appliquée non plus seulement au texte biblique, mais à l'ensemble de l'histoire, jusqu'à l'histqire individuelle. Notre vie, si elle est chrétienne, reproduirait, à notre mesure, les étapes de la vie exemplaire du Christ. La figure de celui-là est alors le chiffre qui donne sens à ce qui n'en a pas sans lui. Voir les travaux de Jacques-Joseph Duguet, Jean-Baptiste d'Etemare. Cf. Bruno NEVEU, «L'érudition ecclésiastique du XVII siècle et la nostalgie de l'Antiquité chrétienne ", in Érudition et Religion aux xvII et xvII' siècles, Paris, Albin Michel, 1994; Henri SAvon, "Le figurisme et la tradition des Pères" , in Bible de tous les temps. T. $6:$ Le Grand Siècle et la Bible, sous la dir. de Jean-Robert Armogathe, Paris, Beauchesne, 1989.

Nous reprenons cette notion en l'adoptant à notre propos. Les fables, ensemble de faits merveilleux et fabuleux, ne prennent sens que si on les réfere, non pas à la seule figure du Christ, mais à l'ensemble de l'Histoire sainte relatée dans la Bible. Le principe herméneutique reste identique : le sens surgit d'une confrontation à une origine que la temporalité ne fait que répéter, en la travestissant, en la déguisant. Aux exégètes et aux érudits d'en révéler la matrice, le modèle figural.
} 
LE FABULEUX, MARQUE DES ORIGINES DE L'ESPRIT HUMAIN

La principale modalité d'analyse du merveilleux, du fabuleux par le père Anselme qu'est le recours au champ de l'erreur, nous le retrouvons également chez Fontenelle dans De l'origine des fables, publié en $1724^{13}$.

Bien entendu, l'étude du fabuleux, et sa caractérisation comme erreur, se fait chez Fontenelle dans un contexte et par rapport à un enjeu qui se distinguent tout à fait de ceux du père Anselme. Mais une chose demeure : le fabuleux, et donc les fables, ne sont pas étudiés en tant que tels; ils ne sont que des erreurs, initiales pour Fontenelle, des balbutiements en quelque sorte. Il n'est pas question de leur accorder une autonomie et une validité signifiantes. L'histoire reste niée au profit d'une classification dont le critère est l'erreur ou la vérité. Le projet de Fontenelle est radicalement différent de celui du père Anselme. Mais il ne donne pas pour autant lieu à une conception proprement historique de la temporalité. Le fabuleux reste une erreur.

Le texte de Fontenelle se propose en fait, à vrai dire, d'étudier les causes, les origines des fables. Mais le début du texte nous intéresse. Il part, en effet, de la fréquentation habituelle des fables grecques, pour montrer que seule une telle habitude nous permet de ne pas rester prisonniers « des amas de chimères, de rêveries et d'absurdités » qu'elles renferment. Nous retrouvons cette ambivalence de la fable: parcours obligé de la culture, mais aussi, et toujours, signe d'un fabuleux qui dérange. Et sur lequel il faut se prononcer.

Nous nous trouvons confrontés à «l'une des plus étranges productions de l'esprit humain ». Le fabuleux révèle cette étrangeté que seule l'habitude nous a rendue familière. Les «esprits follement amoureux de l'Antiquité », cherchant à justifier à tout prix leurs amours, y chercheront "les secrets de la physique ". Il est une autre manière de concilier habitudes culturelles et rationalisme moderne : «Recherchons donc autre chose dans les fables que l'histoire des erreurs de l'esprit humain. » L'Antiquité et ses «productions » fabuleuses, étranges se trouvent ainsi annexées dans une histoire de l'esprit humain. Et comme elles en occupent la première place, elles en représentent le premier stade.

Nulle révocation a priori chez Fontenelle. Les fables peuvent nous apprendre quelque chose, y compris le fabuleux. Car là réside justement

13. Nous ferons référence à sa réédition à Paris en 1990. 
leur signification : c'est parce qu'elles sont fabuleuses que les fables nous intéressent. Nous assimilons le fabuleux à l'erreur, à l'ignorance, qui en même temps lui assignent une place dans le développement de l'esprit humain.

Les fables ne renvoient donc qu'à l'ignorance. Le fabuleux, ce merveilleux, cet « amas de chimères » qu'elles renferment, n'est pas analysé en tant que tel, mais référé à la vérité, qui se trouve être, non plus la Bible, mais le développement actuel de l'esprit humain. Seule l'ignorance, et l'erreur qu'elle produit, donne une place aux fables. Celles-là ne sont par conséquent toujours que la manifestation de l'erreur.

Mais dans le même temps, il faut remarquer que l'Antiquité est légitimée. Elle représente tout de même l'origine, non pas de l'histoire, mais du développement d'une structure qui échappe au temps, l'esprit humain. Celui-là ne s'est pas trouvé d'emblée développé. Il a commencé par prendre une forme grossière, balbutiante. C'est cette première étape, cette origine que représente l'Antiquité. Il faut remarquer que l'origine n'a pas du tout la même fonction que chez le père Anselme. Pour Fontenelle, elle n'est nullement substantielle; elle ne représente pas le foyer de sens, pleinement développé, à partir duquel se déroulerait une temporalité à qui elle donnerait son sens. Elle n'est que le premier stade d'un développement, dont l'époque de Fontenelle est l'aboutissement. La temporalité ne prend plus sens par rapport à l'origine, elle est orientée vers le présent, qui la développe et l'exprime le mieux. À tel point qu'il est possible de comparèr, de trouver " une conformité étonnante entre les fables des Américains et celles des Grecs ». Finalement, espace et temps sont deux modélisations équivalentes d'une même variation structurelle, qui représente l'émergence et l'affirmation de la raison.

Lire les fables de l'Antiquité ne renvoie donc nullement à un projet historique qui chercherait à saisir le sens, la signification de cette époque. S'intéresser aux fables, remarquer le fabuleux qu'elles renferment s'intègre dans une étude génétique, parce que structurelle, de l'esprit humain. L'apparent paradoxe de Fontenelle réside dans ce rapport entre un point de vue génétique (il s'agit bien de décrire un développement dont il se propose d'examiner l'origine, le premier terme et sa manifestation' qu'est le fabuleux), et un point de vue structural (il existe bien une nature de l'esprit, qui se dévoile à travers diverses étapes dont la logique de succession est bien moins chronologique que guidée par le critère de vérité). Les critères de l'erreur et de la vérité sont dès lors requis, mais aiguillent l'étude non vers l'histoire, compréhension d'une époque, mais vers l'explication, dont la logique n'est pas temporelle, des étapes de l'esprit commun. Fontenelle parle d'ailleurs de développement de l'esprit humain et non d'histoire.

Dès lors que l'on utilise les notions de vérité et d'erreur pour expliquer les fables, on s'interdit de comprendre le fabuleux. Celui-là n'est que le 
signe, le moment d'un développement dont la logique lui est étrangère. Le fabuleux est là pour renvoyer à quelque chose d'autre que lui-même, et qui lui donne son sens. Qu'il s'agisse de la Bible ou du développement de la raison, le sens du fabuleux est indépendant.

Ce n'est pas l'histoire, reconnaissance de la compréhension/reconnaissance d'une herméneutique autonomisante du sens propre de chaque société historique, qui pourra faire comprendre le fabuleux. Le temps est résorbé dans une logique, dans une explication qui renvoie ailleurs, vers la Bible ou la raison. Dans cette perspective, l'histoire (ou les événements historiques ou la temporalité) a au mieux une signification négative : être le reflet négatif, inversé d'une vérité atemporelle.

La temporalité ne deviendra histoire que lorsque l'on reconnaîtra que chaque époque crée son propre univers significatif. C'est-à-dire lorsque chaque époque ne sera plus jugée par rapport à une vérité indépendante du temps. C'est-à-dire, enfin, lorsque le fabuleux ne sera plus le contraire ou la déformation d'une vérité anhistorique, mais le reflet de sa propre époque.

LE FABULEUX, MARQUE DE « LA TREMPE DU GÉNIE DES GRECS »

Avec Nicolas Fréret, c'est une toute autre manière d'envisager les fables qui apparaît. Les mots, là encore, sont révélateurs : N. Fréret, s'il continue dans certains textes d'employer la notion de fable, emploie plus volontiers et plus souvent la notion de mythologie. En particulier, dans le titre du texte que nous allons lire : "Réflexions générales sur la nature de la religion des Grecs et sur l'idée que l'on doit se former de leur mythologie ${ }^{14}$.» En passant de la fréquentation des fables à l'étude de la mythologie, N. Fréret change radicalement leur enjeu et présente une pensée de l'histoire.

Dans ce texte, à nouveau, N. Fréret part d'une constatation propre à son époque : «[...] l'étude mythologique est nécessaire aux arts. 》 La production culturelle du $\mathrm{XVIII}^{e}$ siècle reste étroitement dépendante des références mythologiques. Mais ce même siècle est soucieux, comme nous l'avons déjà remarqué, de contrôler le merveilleux, le fabuleux caractéristique de cette mythologie. Il s'est souvent contenté d'une réduction du merveilleux au naturel. Ce procédé, que l'abbé Antoine Banier, autre figure célèbre de l'Académie des inscriptions et belles-lettres, a pratiqué, consiste à « dépouiller les faits de cette espèce d'un merveilleux souvent obscur », à

14. Publié au tome XXIII des Histoires de l'AIBL, Paris, 1756. 
« réduire les dieux au simple rang des héros et les héros au rang des hommes ». Les fables, pour cet évhémérisme moderne, ne sont que le reflet de l'histoire antique, des événements qui en constituent la trame ${ }^{15}$. Or cette réduction n'est pour N. Fréret, pas satisfaisante, parce qu'incomplète. «Cette clé n'est pas la seule ni la plus importante » pour comprendre la mythologie. Cette critique de l'évhémérisme d'A. Banier détermine la façon dont N. Fréret et le chevalier de La Barre ${ }^{16}$ vont interpréter le merveilleux. Il ne peut être considéré uniquement comme une déformation d'événements historiques, par ailleurs connus positivement. Le merveilleux, le fabuleux, ne peut être réduit de la sorte. Apparemment, une séparation entre mythologie et histoire est la conséquence immédiate de cette critique. Il n'est pas possible d'aller chercher la vérité au-delà du merveilleux, qui ne serait plus alors qu'un artefact de cette vérité, et qui perdrait tout intérêt dès lors qu'elle serait atteinte. Le fabuleux ne se réduit pas si facilement. Et l'éloignement de la mythologie vis-à-vis de l'histoire n'est à comprendre que comme éloignement de ce rapport direct que le merveilleux entretiendrait, selon A. Banier, avec des événements historiques.

N. Fréret précise, en effet, que l'on peut envisager « la mythologie [et le fabuleux qu'elle rencontre] comme l'introduction à l'histoire de l'Antiquité ». Car les « anciennes fables renferment la religion », selon le chevalier de La Barre. N. Fréret souscrit à cette même thèse, et en donne plusieurs illustrations dans différents Mémoires à l'Académie des inscriptions et belles-lettres ${ }^{17}$. Les fables sont une allégorie de la religion. De telle sorte qu'en les étudiant, on " y découvre la trempe du génie naturel des Grecs ». Étudier les mythes grecs ne se fera plus par rapport à une vérité, qui les désignerait en retour comme erreur ou déformation. Il ne saurait donc plus s'agir de procédés de réduction ou de dérivation afin de les étudier.

La méthode que proposent N. Fréret et le chevalier de La Barre est ipso facto déconnectée de tout enjeu de vérité. Il n'est plus question d'établir l'erreur (par rapport à la Bible) ou la déformation (par rapport à un événement) du merveilleux grâce à une logique de dérivation ou à un principe de

15. Jean SEznec, in La Survie des dieux anciens à la Renaissance, Paris, Flammarion, 1993, offre une présentation des diverses modalités d'interprétation des fables et des mythes de l'Antiquité durant la Renaissance, modalités qui sont remobilisées au XvII' siècle.

16. Compagnon de N. Fréret à l'AIBL, dont ce dernier publiera les « Mémoires pour servir à l'histoire de la religion de la Grèce » au tome XVI des Mémoires de l'AIBL, Paris, 1751. $\mathrm{N}$. Fréret continuera ses travaux, en particulier dans le sens d'une critique de l'évhémérisme, dont l'abbé A. Banier était le représentant principal à l'AIBL.

17. On pourrait citer parmi les Mémoires de l'AIBL: «Observations sur le temps auquel a vécu Bellérophon », 1733; « Réflexions sur les prodiges rapportés par les Anciens », 1717; "Remarques sur les fondements historiques de la fable de Belléphoron, et sur la manière de l'expliquer ", 1733; «Recherches sur le cycle de Bacchus parmi les Grecs », 1749; « Recherches pour servir l'histoire des Cyclopes, des Dactyles, des Telchines, des Curètes, des Corybantes et des Cabires », 1756. 
réduction. Il s'agit bien plus d'en établir une carte, qui nous permette éventuellement d'en saisir le sens.

Nous pouvons préciser la méthode qui sera celle du mythologue, telle que la décrit $\mathrm{N}$. Fréret. Face à chaque mythe, face à chaque détail fabuleux, il faut être attentif aux lieux et aux circonstances. En effet, les mythes constituent autant « de combinaisons [qui] se diversifi[ent] à l'infini suivant les lieux et les circonstances ». À cette condition, on pourra voir surgir un jeu de «combinaisons", une multiplicité que tout esprit de système viendrait écraser, un « corps informe et irrégulier ».

La mythologie doit donc éviter la réduction afin de conserver vivante cette multiplicité. Étudier le merveilleux revient donc à dénombrer des combinaisons. Son rôle s'arrête là; l'historien peut alors intervenir, en espérant en faire surgir une combinatoire, logique, qui en donnerait la clé. L'historien en est d'ailleurs tout à fait capable, si l'on sait quelles doivent être ses propres qualités selon $\mathrm{N}$. Fréret ${ }^{18}:$ l'attention à la spécificité et à la particularité de chaque information et la capacité d'opérer des liaisons entre ces informations dont il peut se servir. C'est à lui que revient donc la tâche de constituer les liens desquels devraient sortir l'ordre et la signification de ces mythes.

Ainsi l'intérêt porté au fabuleux, qui n'exclut pas qu'on puisse le trouver "souvent absurde», doit permettre de saisir «le génie national des Grecs ». Le fabuleux, les mythes, au-delà de ce qui peut choquer notre sens commun, nous fait entrer en rapport avec le génie, les mœurs propres à une époque. Cet intérêt porté à ce qui unit indéfectiblement les divers membres d'une communauté, et que l'on peut saisir de diverses manières, ne naît pas avec $\mathrm{N}$. Fréret. La deuxième partie du xvir ${ }^{e}$ siècle a vu s'opérer le changement de registre d'une notion d'abord épistémologique (le «bon sens » cartésien) avant, avec Charles de Saint-Évremond en particulier, de recouper un champ historique et sociologique. L'interrogation sur le sens commun, sur le génie propre à chaque peuple («[...] chaque peuple avait ses dieux et ses lois et ses coutumes») est également tributaire de la critique que Pascal mène quant à l'universalité des notions communes et du sens commun ${ }^{19}$.

Le génie d'un peuple n'est plus étudié par rapport à un critère de vérité; mais il permet de décrire les combinaisons spécifiques propres à chaque

18. Pour une vue plus précise des conceptions méthodologiques de N. Fréret, voir « Vues générales sur l'origine et le mélange des anciennes nations, et sur la manière d'en étudier l'histoire », Histoires de l'AIBL, t. XVIII, Paris, 1753.

19. Une étude des notions de bon sens et de sens commun mettrait en évidence ce basculement, dont l'enjeu final apparaîtra au XVIII' siècle. À travers la reconnaissance pleine et entière, chez Vico, Montesquieu, Voltaire ou encore Herder, de la notion de sens commun. Il ne saurait plus être question alors de critiquer le sens commun d'un peuple au nom de la raison; celle-là n'apparaît jamais qu'à travers les différentes modélisations particulières, mais toutes équivalentes, qu'elle prend tout au long de l'histoire. 
société. Et, dans cette entreprise, l'analyse du fabuleux est pour N. Fréret la première étape inévitable et irremplaçable, comme l'atteste le nombre de ses communications consacrées aux mythes grecs, romains et à d'autres peuples de l'Antiquité.

La saine critique doit par conséquent « envisager [la mythologie] comme l'introduction à l'homme de l'Antiquité ». Étudier les mythes grecs ne se fera plus dans un rapport avec une vérité qui les désignerait en retour comme erreur ou déformation. Il ne saurait plus s'agir de dérivation ou de réduction. Les mythes possèdent une signification qui nous fera accéder à l'homme de l'Antiquité. Nous pourrons y découvrir « la trempe du génie national des Grecs ».

Pour bien comprendre cette relation entre études mythologiques et histoire, il nous faut insister sur la notion de système, de combinaison dans la méthode de $\mathrm{N}$. Fréret. Ce dernier envisage les mythes grecs comme autant de « combinaisons [qui] se diversifi[ent] à l'infini suivant les lieux et les circonstances ». L'uniformité est absente, le merveilleux varie, change en fonction des auteurs qui le rapportent. Un mythe, véhicule du fabuleux, n'est jamais fixe. Ce jeu de combinaisons fabuleuses et merveilleuses produit un « corps informe et irrégulier ». Il faudra par conséquent pour l'analyser être attentif aux circonstances et aux lieux.

La mythologie doit donc éviter la réduction afin de conserver vivante cette multiplicité. Ainsi, étudier le merveilleux revient à dénombrer des combinaisons, en espérant en faire une combinatoire, c'est-à-dire en tirer un ordre. Il faut insister sur le fait qu'une telle méthode est ipso facto séparée de tout enjeu de vérité. Il n'est plus question d'établir la vérité ou l'erreur du merveilleux grâce à des procédés de dérivation ou de réduction; il s'agit d'en établir une carte, qui nous permette d'en comprendre le sens.

Une difficulté surgit lorsque N. Fréret insiste sur le fait que l'étude des mythes n'aboutit pas à l'élaboration d'un ordre mais beaucoup plus à l'étonnement devant une absence apparente de systématicité.

Le chevalier de La Barre souligne l'autonomisation de la mythologie par rapport à la problématique de la vérité. Il remarque en effet combien « [...] il a fallu renverser les fables pour y retrouver l'Histoire sainte [...]. On a usé des fables comme des mots d'une langue pour y exprimer tout ce qu'on veut ». L'allégorie, la nouvelle méthode exposée par N. Fréret, reprenant la critique menée par le chevalier de La Barre de l'évhémérisme d'A. Banier, interdisent dorénavant tout renversement. Le fabuleux n'est plus le simple indice, le simple artefact d'une signification d'une vérité qui se dévoilerait ailleurs. Le fabuleux est le symbole du génie du peuple qui l'a créé.

Cet aspect symbolique du merveilleux, en rapport nécessaire avec l'époque qui le sécrète, un autre membre de l'Académie des inscriptions et belles-lettres le soulignera avec encore plus de détermination dans la 
seconde moitié du xvill ${ }^{e}$ siècle. Il s'agit de Jean Lévesque de Burigny, frère de Louis-Jean Lévesque de Pouilly qui avait brièvement défrayé la chronique académicienne en 1722 en remettant en cause la possibilité même d'une connaissance assurée de l'histoire romaine ${ }^{20}$. Dans une communication intitulée "Sur le goût du merveilleux reproché aux hommes grecs et latins », J. Lévesque de Burigny met d'abord l'accent sur la vanité dont font preuve les nations, qui toutes cherchent à se parer d'origines fabuleuses et merveilleuses. Ces fables «plus ou moins ridicules», dont J. Lévesque de Burigny a rassemblé « une partie des traits aussi fabuleux que merveilleux », ne doivent pas nous rebuter, dès lors que l'on se sera rendu compte quelle peut être leur utilité.

En effet, note cet auteur, si les historiens antiques ne les avaient rapportés, une « lacune que rien ne serait capable de combler » apparaîtrait. Car « nous serions aujourd'hui hors d'état de bien connaître le génie, le caractère, les mœurs des Nations ». Il ajoute que "derrière un préjugé reposait quelquefois une vérité utile ». Si l'on veut connaître une époque, il nous faut en connaître les mœurs, le génie particulier. Et pour approcher ce génie, si différent du nôtre, nous avons impérativement besoin de ses productions, au nombre desquelles figurent en bonne place le fabuleux et le merveilleux pour les Grecs et les Romains. Il n'est plus question de les rejeter ou de les dépasser au nom de la vérité. Ils nous sont utiles; ils représentent une «vérité utile», une vérité propre à la société considérée, expression adéquate de ses «préjugés ».

Cette notion de préjugé n'a évidemment plus son sens épistémologique, critiqué par Descartes. Le préjugé est avant tout l'indice, le symbole encore, d'une société. Il serait dommage de le critiquer au nom d'une vérité dont les prémices nous manquent ${ }^{21}$. L'enjeu de J. Lévesque de Burigny est donc véritablement historique et non plus dogmatique, gnoséologique ou encore apologétique.

Seule importe l'utilité historique du fabuleux, utilité indéniable tant il permet de saisir le génie d'un peuple. La vérité n'est plus ce au nom de quoi il serait possible de rejeter le fabuleux. Celui-là est au contraire la voie d'accès, la seule qui nous soit accessible, menant à la définition du génie d'une époque. Les critères de la vérité et de l'erreur ne sont plus de mise.

20. Les deux frères ont été tous deux en contact avec les cercles voltairiens. Cette relation ne se retrouve pas uniquement dans le pyrrhonisme mesuré dont tous feraient preuve. On peut également noter le rapport entre l'intérêt porté par L.-J. Lévesque de Pouilly aux mœurs et l'étude que Voltaire fait de l'histoire également du même point de vue.

21. Là encore G. VICO, in la Science nouvelle (1725), mais également in La Méthode des études de notre temps (1709), présente une conceptualisation tout à fait intéressante de cette notion. Le préjugé mène en fait au sens commun; et la vérité, qui ne peut être possédée analytiquement, ne pourra être atteinte qu'à partir d'une étude synthétique des sens communs historiques, des diverses formes de préjugés que l'on pourra rencontrer dans l'histoire. 
L'histoire instaure une autre méthode, d'autres notions, parmi lesquelles celle de signification. Chaque époque a sa propre signification, ses mœurs et son génie. Le fabuleux, caractérisation du génie des peuples antiques, représente de ce fait une "vérité utile ", et non plus la déformation d'une vérité anhistorique, dont le critère absolu serait l'adéquation au dogme ou à la Bible, ou encore le bon fonctionnement de l'esprit humain. Il n'y a plus, à vrai dire, de critère absolu au nom duquel condamner, juger ce fabuleux. Nul doute que l'histoire, interprétation des sociétés passées, ne pouvait naître que sur les ruines d'une telle conception judiciaire de l'examen des productions du passé.

Nous avons essayé de mettre en évidence tout d'abord les schémas d'analyse du fabuleux. Il nous a semblé que l'on pouvait y lire un débat quant à la conception de la temporalité. Entre Tradition et Histoire, l'appréciation du fabuleux change inévitablement, passant de la négation à la reconnaissance. Un des signes d'une pensée et d'une pratique de l'histoire passe certainement par cette reconnaissance d'une autonomie herméneutique de chaque société.

Est-ce à dire que la pensée française s'est définitivement convertie à l'histoire au courant du XvIII esiècle? Certainement non. Car nous avons également essayé de montrer que la lutte contre la Tradition pouvait aussi être le fait d'une méthode rationaliste, tout aussi anhistorique que la Tradition et que symbolise Fontenelle. L'Histoire trouve alors un nouvel adversaire, qui la combattra, d'une nouvelle manière, et selon d'autres enjeux. L'Encyclopédie sera, de même que le milieu érudit français, le lieu et le témoin de ce nouveau débat. 